nobles of the Winter Queen's court, with dancing until 4 a.m., or walking through the estate garden of the elder Huygens, or accompanying his third son Lodewijk through Somerset House in London after the execution of Charles I to view the impressive royal art collection.

This fascinating book is an excellent introduction to seventeenth-century Anglo-Dutch relations. Jardine does not try to summarize the whole field. She avoids, for instance, examining the Anglo-Dutch wars of the period or the bitter rivalries abroad, gives much more space to royalists than republicans, scarcely deals with religion, and treats the formative period of English philosopher John Locke's exile in the Netherlands very lightly. She does not develop fully an account of how the Dutch coup launched the Bank of England, nor how it affected Scotland and Ireland. These subjects are left to other works, many of them cited in her bibliography. Jardine presents the view from England more than that from across the North Sea, and her subtitle is perhaps more relevant to the eighteenth century than the seventeenth. Yet by exploring pertinent examples, Going Dutch demonstrates that personal connections helped to shape the cultures of both countries.

Harold Cook is professor at and director of The Wellcome Trust Centre for the History of Medicine at University College London, London NW1 2BE, UK, and author of Matters of Exchange: Commerce, Medicine and Science in the Dutch Golden Age.

\title{
Biased brains, messy memories
}

\author{
Kluge: The Haphazard Construction of the \\ Human Mind \\ by Gary Marcus \\ Houghton Mifflin: 2008. 224 pp. \$24
}

\section{A Portrait of the Brain \\ by Adam Zeman \\ Yale University Press: 2008. 256 pp. \\ $\$ 27.50$}

\section{Sandra Aamodt}

Public interest in the brain seems to be insatiable, judging from the many popular books about the topic that have been published in the past few years. Highlighting the diversity of this expanding genre, two books aimed at general readers provide views of brain science in very different styles.

In Kluge, psychologist Gary Marcus presents a lively tour of the shortcomings of human minds and concludes that evolution has left us with something of a mess. In an argument reminiscent of David Linden's The Accidental Mind, Marcus makes his case by describing cognitive difficulties, including false beliefs, linguistic ambiguity, impulsiveness and mental illness.

The blame, he asserts, rests with our imperfect memory, "arguably the mind's original sin". Perhaps we would reason more effectively if the brain could store and retrieve data as accurately and as simply as a computer. Instead we must contend with a limited system. Brains locate memories by matching them to the current context rather than having unbiased access to all of our experiences. This contextual dependence makes it hard during an argument, for example, to recall how often our spouse does the housework, because thinking of one failure inclines our brains to remember similar situations rather than contrary examples.

Many of these problems result from conflicts between the brain's two basic styles of thinking.
The reflexive system, having evolved earlier, controls most behaviour. It is fast and can accurately assess statistics - such as the likelihood of finding food in certain locations - but is prone to overgeneralization and snap judgements. The deliberative system, by contrast, is slow, effortful and logical, at least intermittently. The reflexive system readily overrides the deliberative system, especially when we are tired or rushed. Marcus believes our lives would be improved if we engaged the deliberative system more often, although he acknowledges that "it often settles for reasoning that is less than ideal".

Evolutionary psychology has tempted many scientists to indulge in just-so stories, as Marcus notes; asserting that our brains are poorly engineered is an equally risky business. Computer memories are more factual than those of humans, but computers lag far behind the reflexive system on other problems, such as distinguishing cats from dogs. Whether any biological system (or a computer) could combine both sets of virtues remains an open question.

Marcus closes with experimentally verified ways for people to deliberate more effectively, a welcome change from the usual self-help prescriptions. For example, he suggests imagining that your decisions might be checked by someone else. Although few biologists will need to be convinced of the evolutionary arguments in this book, it remains intriguing to contemplate which aspects of our minds could be improved and how we might compensate for those weaknesses.

A Portrait of the Brain is constructed with equal care, but in a very different style. Neurologist Adam Zeman takes on an ambitious project: explaining brain function from atoms to neural networks to, unexpectedly, the soul. Clearly an Oliver Sacks fan, Zeman weaves case studies of patients together with basic science, history, etymology, classical literature and art to produce an erudite discourse on brain components.

Describing the many aspects of neurons, the fifth chapter explains, for instance, how early neuroanatomists determined that brains are composed of cells, tells the story of a bus driver with religious feelings caused by epileptic seizures who ultimately became a priest, and is laced with asides about scientific rivalry, brain development, the hobbies of neuroscientist Santiago Ramón y Cajal and how action potentials are generated. The science is clear, and the stories of patients are suspenseful and gripping. Yet in some places, the relationship between topics feels a bit forced.

In the final chapter, Zeman grapples with consciousness. He outlines how brains that are predisposed to tell stories and that attribute actions to agents rather than chance might lead us to believe in an immortal soul. His own view is that this is "no more than a wonderful fiction". (Marcus makes the same point less gently.) Zeman struggles with science's failure to find an emotionally satisfying replacement story, conceding that such questions may be more in the realm of art than science.

What is the impetus for this deluge of brain

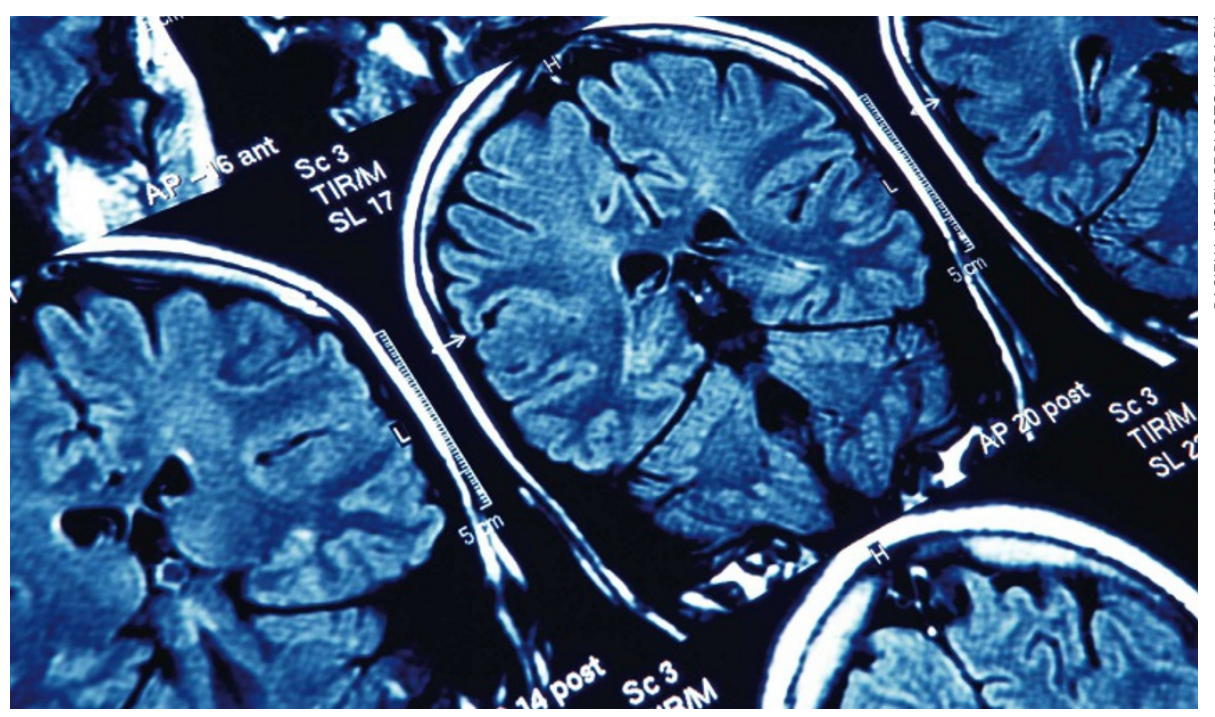

Powers of persuasion: human brain images have piqued public interest in neuroscience and psychology. 
books? Applications outside of science may explain the popularity of some topics, such as the potential for behavioural economics to illuminate the decisions of investors. Brainscan images themselves may also have amplified interest in neuroscience. Both the public and the media are drawn to the powerful and persuasive visual message of such images.
Fundamentally, however, people remain interested in neuroscience and psychology because understanding our brains helps us to understand ourselves.

Sandra Aamodt is former editor of Nature Neuroscience and author of Welcome to Your Brain: Why You Lose Your Car Keys But Never Forget How to Drive and Other Puzzles of Everyday Life. computation. Classifications based on gene sequences are hugely disruptive to the established order of doing things. The focus of Systematics as Cyberscience is not rapid genetic taxonomy, which could fill its own book, but these recent advances only heighten the identity shift now under way in taxonomy.

As taxonomy evolves into the cyber age, it is reappearing in the public eye. In February 2008, systematists launched the first digital version of the Encyclopedia of Life (www. eol.org), wherein many of the approximately 1.8 million known species on Earth will be represented, each on its own web page. The fact that most of these pages are still blank does not dilute the mythical power of this embryonic encyclopaedia. We can see its promise in the 30,000 populated pages now in operation. Each species page includes photographs of the organism in its various life stages, the original taxonomic description, updatable geographical maps of its range, classification charts, links to ecological niches, popular knowledge of the organism, and many other attributes needed to understand its biology.

The most important aspect of this emerging cyber library is the way in which it facilitates the completion of the great taxonomic imperative: to identify all of the living species on Earth. There is doubt as to how long this will take, but it will surely happen only with the help of vast communication webs and computer technology.

The future of systematics will involve advances in computational photography, innovations in creating identification keys, pattern-recognition software, expert-knowledge

The Encyclopedia of Life will log facts for all species on Earth. , and remains, parochial. The exper tise required for classifying fly parasites has little in common with that for fungal species or whales. Taxonomic information occupies niches - niche being the exact biological term for these narrow confines. Specialized niches of information with their own protocols challenge computerization.

Third, the low priority given to taxonomy has meant it is perennially underfunded. Highpowered computation and software come low on the list after the meagre needs of traditional taxonomy are (barely) met.

Despite these hurdles, the related field of systematics (exploring relationships between organisms over time) is rapidly transforming itself as computation becomes integral. In Systematics as Cyberscience, sociologist Christine Hine investigates the effects of computers and communication technology on the taxonomic community.

Hine's subjects are primarily the practising taxonomists and systematists in the United Kingdom, but this community is representative of that in the rest of the world. Her concern is the sociology of change, how these tradition-bound disciplines imagine, place and incorporate computers into their identity.
For instance, she notices that "the Web and the Internet are seen ... as evocative objects which bring a certain glamour and an image of modernity and accessibility to systematics". Computer technology is not only an aid to getting work done, and to getting new kinds of work done, but also to securing the respect and funding needed to do any work at all. This strategy plays into a misperception that you are not doing real science unless you are doing it on computers.

As a consequence of digitalization, classical taxonomy is put under different strains as it adjusts to its new tools. Hine notes that: "Traditionally, systematics has worked on long time scales, with taxonomists focusing on grand life works, and major flora projects taking maybe 30 years to complete." Now, by contrast, taxonomy projects based on Internet databases are offered up in minutes, and are never viewed as finished. Instead they remain in the 'perpetual beta' state of most digital content.

Cheap, ubiquitous genetic sequencing is further accelerating taxonomy's rush into systems and, eventually, genetic identification gadgets. Soon, the practice of taxonomy will be unthinkable without the Internet. Yet, as Hine notes, at the moment "systematics has been able to portray itself as fundamentally unchanged by its experience with these technologies, stressing instead the way it is pursuing its established goals and preserving its heritage."

The long sweep of evolution breeds in those who try to untangle it a great appreciation of the winnowing process of time. Taxonomists are wary of tossing away any highly evolved method of knowledge that has proven itself. It is remarkable then to see the speed at which these refined practices are being reimagined for this digital century.

Systematics as Cyberscience reminds us that change generates strain, conflicting views, fear of the new and concern for integrity. But we cannot stop this shift that is being played out by experts in their respective museums and natural history collections.

Kevin Kelly is senior maverick at Wired magazine and chair of the All Species Foundation. 